\title{
An Aerodynamic Extension for Motion Planning with Dynamics Awareness in Aerial Long-Reach Manipulators
}

\author{
Alvaro Caballero $\mathbb{D}^{1},{ }^{1}$ Pedro J. Sanchez-Cuevas, ${ }^{1}$ Manuel Bejar, ${ }^{2}$ Guillermo Heredia, ${ }^{1}$ \\ Miguel A. Trujillo, ${ }^{3}$ and Anibal Ollero ${ }^{1}$ \\ ${ }^{1}$ GRVC Robotics Lab., University of Seville, Camino de los Descubrimientos S/N, 41092 Seville, Spain \\ ${ }^{2}$ GRVC Robotics Lab., University Pablo de Olavide, Ctra. Utrera Km. 1, 41013 Seville, Spain \\ ${ }^{3}$ CATEC (Advanced Center for Aerospace Technologies), C/Wilbur y Orville Wright 19, 41309, La Rinconada, Seville, Spain
}

Correspondence should be addressed to Alvaro Caballero; alvarocaballero@us.es

Received 21 February 2020; Revised 5 August 2020; Accepted 24 August 2020; Published 19 September 2020

Academic Editor: Luis E. González-Jiménez

Copyright (c) 2020 Alvaro Caballero et al. This is an open access article distributed under the Creative Commons Attribution License, which permits unrestricted use, distribution, and reproduction in any medium, provided the original work is properly cited.

\begin{abstract}
This paper presents a novel method for motion planning of aerial long-reach manipulators that considers the aerodynamic effects generated by close surfaces in the trajectory generation process. The aerial manipulation system consists of a multirotor equipped with a robotic long-reach arm that enables multidirectional inspection and also increases considerably the safety distance between the rotors and the inspected elements. Since these systems operate in the proximity of elements that can modify significantly the rotors' airflow, the inclusion of Aerodynamics Awareness within the motion planning process is required to ensure robust obstacle avoidance. To this end, a proper characterisation of the aerodynamic effects based on both theoretical and experimental considerations has been derived. This characterisation is taken into account in the trajectory generation process to discard states whose associated aerodynamic phenomena are not well compensated by the system controller and to explore alternatives that lead to the most efficient trajectories within the area of safe operation. Moreover, the motion planner also stands out for three other relevant features: the joint consideration of the multirotor and the robotic long-reach arm, the generation of efficient trajectories in terms of energy consumption, and the Dynamics Awareness of the strong coupling between the aerial platform and the robotic arm. The resulting motion planner has been successfully tested in a simulated environment that faithfully reflects an application scenario strongly affected by aerodynamic effects: the inspection of bridges to find potential cracks in the surface of pillars.
\end{abstract}

\section{Introduction}

Among the numerous applications in which Unmanned Aerial Vehicles (UAVs) can be used, aerial manipulation is arousing much interest. Potential applications in this field include instrument deployment, maintenance operation, and contact inspection in industrial sites or civil constructions in which access is very dangerous or costly. The motivation is to decrease risks and operational costs. A small-size rotorcraft can indeed access hard-to-reach places more easily than human operators, avoiding unnecessary risks for industrial workers and allowing inspection and maintenance operations without shutdowns of the facilities (the mandatory safety policy in case of human operation) and without the use of scaffolding or cranes.

These new promising applications of aerial robotic systems for manipulation tasks bring also new challenges. Firstly, it is necessary to develop new manipulation or inspection tools, such as adapted arms, which can be seamlessly integrated into the airframe. Furthermore, the existing algorithms to operate the UAV and the manipulators autonomously should be extended for the integrated system. In this respect, one of the most challenging issues is the development of new methods that consider both the UAV and the manipulator dynamics when planning the motion of the complete aerial manipulation system. When moving between 
different locations inside a dense industrial installation or close to obstacles, this planning will be essential for the generation of accurate and collision-free movements.

Many research works about aerial manipulation have been recently published. [1] presents the design of several lightweight, low-complexity grippers that allow quadrotors to grasp and perch on branches or beams and pick up and transport payloads. Additionally, in [2], a human-size and lightweight dual-arm manipulator is integrated in a multirotor platform and tested in outdoor flights. On a very different system scale, [3] proposes a system for aerial manipulation, composed of a helicopter and an industrial manipulator. In other valuable contributions [4, 5], a multirotor with a robotic arm attached to its top part is developed for inspection tasks on bridge ceilings. In view of simulation, [6] focuses on the derivation of the dynamic model of a hexacopter equipped with a robotic arm and the analysis of its stability. For industrial applications, [7] presents a novel aerial robotic manipulator that provides contact capabilities for inspection and maintenance. With similar purposes, [8-11] introduce the usage of long-reach aerial manipulators. These systems consist of an aerial platform endowed with a longbar extension that incorporates a lightweight manipulator in the tip. This configuration allows aerial manipulation tasks even in hard-to-reach places since it increases considerably the safety distance between rotors and manipulated objects.

However, although a large number of works have focused on the development of control techniques for the system integrating the aerial vehicle and the manipulator devices, not many of them deal with the associated motion planning problem. Furthermore, the existing contributions like [12] usually assume a strong simplification by addressing the planning problem in a decoupled way, i.e., adopting independent planners for the UAV and the manipulators that swap their operation according to the mission phase. Contributions where the motion planning of the vehicle and the manipulator is addressed integrately are mainly limited to manipulators mounted on mobile ground bases. As an example, [13] deals with this problem on flexible mobile manipulators which can be modelled as in [14]. In contrast to previous works, the authors of this paper presented in [9] a novel algorithm that considers jointly the aerial platform and the manipulators within the planning operation. This integrated strategy, validated through simulation and experimentation in [11], allows the consideration of wider and safer operating conditions.

There also exist some contributions that present planning methods where explicit consideration of dynamics is required to generate admissible and collision-free trajectories. Thus, [15] proposes kinodynamic motion planners that consider kinematic and dynamic constraints during trajectory generation. However, these planners require a planning space twice the dimension of the configuration space-configuration variables together with their associated velocities-which implies high computational costs. Alternatively, [16, 17] propose other methods that split the motion planning problem into two stages. In the first stage, a basic planner searches for a path compatible with a bounding sphere that replaces the system. In the second stage, the path is transformed into a trajectory compatible with the kinodynamic constraints. A significant drawback of these methods comes from the approximation required in the first stage since it could complicate the existence of a collision-free path. Along with these works, the authors of this paper presented an extension of [9] in [10] to include Dynamics Awareness within the operation of the planner for robust obstacle avoidance. To this end, the expansion of the search tree is based on closed-loop simulations of the controlled aerial manipulator instead of using geometrical interpolations.

Another aspect that should not be neglected when planning the motion of an aerial system is the hazardous influence of the aerodynamic effects associated with the operation of rotors in cluttered environments. Indeed, the airflow generated by rotary-wing platforms is very influenced by the physical obstacles close to it. For this reason, the proximity of surfaces like the ground or a ceiling disturbs the natural motion of the air considerably, causing significant changes in the thrust generated by the rotors of the aerial platform. These aerodynamic phenomena, known, respectively, as ground effect and ceiling effect, can be dangerous for standard multirotors provoking their destabilisation or even crashes. To the authors' knowledge, there is not any precedent contribution in the literature that addresses these undesired effects of the aerodynamic phenomena at the planning level. Instead, there are some contributions that deal with the aerodynamic phenomena at the control level. In this way, [18] proposes an enhanced control scheme for quadrotors with the purpose of improving the landing under the presence of the ground effect. Moreover, [19] analyses the influence of the ground effect in several control approaches for multirotors. Additionally, [20] proposes a new UAV configuration that takes advantage of the ceiling effect. The latter allows precise contact inspection on bridges to be carried out.

This paper extends the previous contribution [10] presented by the authors, where Dynamics Awareness (DA) is firstly introduced for robust obstacle avoidance. The most relevant extension is the consideration of aerodynamic effects within the motion planning process to complement the Dynamics Awareness approach. This new formulation implies that the expansion of the search tree will be based not only on the dynamics of the controlled system but also on its aerodynamical behaviour. To this end, a proper characterisation of the aerodynamic effects based on both theoretical and experimental considerations has been derived. This characterisation is considered within the trajectory generation process to discard states whose associated aerodynamic phenomena may provoke undesired collisions and to explore alternatives that lead to the most efficient trajectories within the area of safe operation. To the best of the authors' knowledge, this paper presents the first motion planner with Aerodynamics Awareness (ADA) to overcome the undesired effects previously described.

As an illustrative example of the potential benefits of the Aerodynamics Awareness, Figure 1 shows the difficulties of flying under the aerodynamic phenomena associated with a cluttered scenario. As can be seen, the UAV tries to follow the straight trajectory in dashed light grey that has been 


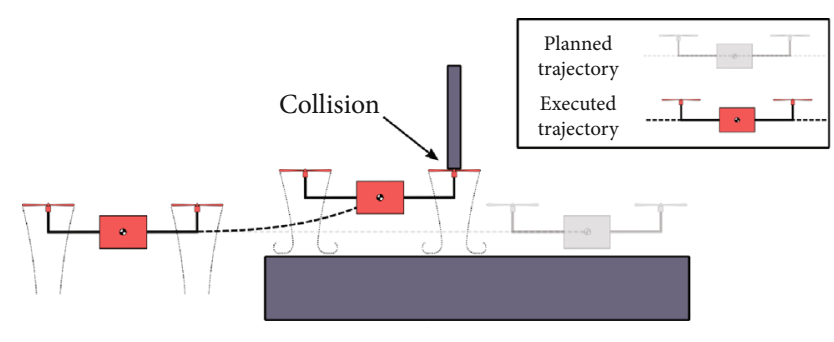

FIGURE 1: UAV trying to track a straight planned trajectory between two obstacles. The system crashes with the upper obstacle as a consequence of an undesirable ascent caused by the ground effect.

generated using a standard motion planner to go through the two dark grey elements considered in the figure. However, due to the ground effect appearing when the system flies close to the lower dark grey surface, an undesirable ascent takes place, and the system collides with the upper dark grey element.

There are several applications that can benefit from this Aerodynamics Awareness. Industrial scenarios like oil and gas facilities (see Figure 2(a)) are a clear example since the existence of large pipe arrays and bulky tanks can provoke aerodynamic interaction. This work proposes the realistic application of bridge inspection as use case to evaluate the proposed motion planner. With the means currently available, the application scenario of crack detection in reinforced concrete bridges (see Figure 2(b)) requires visual inspection by qualified human operators. This inspection usually demands the usage of scaffolding or cranes in hard-to-reach and high-altitude locations, which endangers human lives.

In that context of the bridge inspection scenario, this paper suggests the usage of a novel Aerial Robotic System for Long-Reach Manipulation (ARS-LRM) in order to avoid human risks and to minimise the operational costs. This aerial manipulator consists of a multirotor and a robotic long-reach arm whose special integration with the aerial platform enables $360^{\circ}$ rotation within the vertical plane which contains the centre of mass of the multirotor. This special configuration of the manipulator allows multidirectional inspection to be addressed and also increases considerably the safety distance between the rotors and the inspected areas, which makes this platform a very convenient solution for the aforementioned scenario of bridge inspection. Additionally, since the ARS-LRM system will have to operate close to the bridge deck, aerodynamic phenomena, such as the ceiling effect, will play an important role. Consequently, this scenario will be an excellent test bench to analyse the benefits associated with the addition of Aerodynamics Awareness within the trajectory generation process.

The rest of this paper is organised as follows. Section 2 describes the ARS-LRM system, the corresponding multibody dynamical model, and the distributed control approach derived for the system. Then, Section 3 focuses on the characterisation of the most relevant aerodynamic effects. In Section 4, the planning algorithm is presented with special attention to the new Aerodynamics Awareness (ADA) mechanism proposed in this paper. In order to better illustrate its benefits, Section 5 defines a realistic scenario to test the algo- rithm, a bridge inspection application. After the presentation of the complete system as well as the motivating scenario, Section 6 includes several closed-loop simulations of the controlled ARS-LRM system when following the planned trajectories given by the different motion planning algorithms that have been considered in this paper. The latter illustrates how the motion planner with Aerodynamics Awareness outperforms the results obtained with algorithms that do not include this feature. Finally, Section 7 is devoted to conclusions and future work.

\section{The Aerial Long-Reach Manipulator for Multidirectional Inspection}

2.1. System Description. As can be seen in Figure 3, the proposed Aerial Robotic System for Long-Reach Manipulation (ARS-LRM) consists of a multirotor and a robotic longreach arm whose special integration with the aerial platform enables $360^{\circ}$ rotation within the vertical plane that coincides with the centre of mass of the multirotor. This capability for multidirectional movement within the vertical plane widens significantly the inspection workspace of the robotic arm, which in turn extends the range of positions from which the aerial platform can perform the inspection task. The latter allows a better selection of the safest operation conditions for the aerial system. Additionally, the long-reach feature increments the safety distance between rotors and inspected areas. This last characteristic is even more relevant when the inspected areas include elements that can affect the rotor airflow, as is the case of the application scenario for this research work. Both features, multidirectional and long-reach capabilities, constitute an important improvement with respect to state-of-the-art aerial manipulators where the base of the arms is usually fixed at the bottom part of the airframe. Moreover, by simply interchanging the tool located as end effector, the ARS-LRM system is endowed with the capability of carrying out a wide variety of manipulation tasks.

It has been shown in the AEROARMS project [22] that a planar characterisation of the system is enough for motion planning. This simplified approach eases the modelling and control derivations while maintaining the operation basis of the system. Following this assumption, the aerial platform $A$ is characterised by a mass $m_{a}$, a principal moment of inertia $I_{22}^{a}$, and dimensions $w \times h$. Concerning the location of the rotors, a quadcopter configuration with propellers of radius $R$ can be selected. In this configuration, the rotors are symmetrically located with respect to the UAV centre of mass $A^{O}$ at longitudinal and vertical distances $d_{1}$ and $d_{3}$, respectively. Additionally, the robotic $\operatorname{arm} B$ is assumed to be aligned with the UAV centre of mass. This long-reach manipulator has been considered as a rigid body of length $l_{b}$, mass $m_{b}$, and principal moment of inertia $I_{22}^{b}$. Its centre of mass $B^{O}$ is located at a distance $\lambda_{b} l_{b}\left(\lambda_{b} \in[0,1]\right)$ with respect to $A^{O}$. Finally, the end effector $E$ has been treated in this simplified model as a punctual mass $m_{e}$ located at the distal end of the robotic arm. The values of the parameters that have been previously defined are shown in Table 1. 


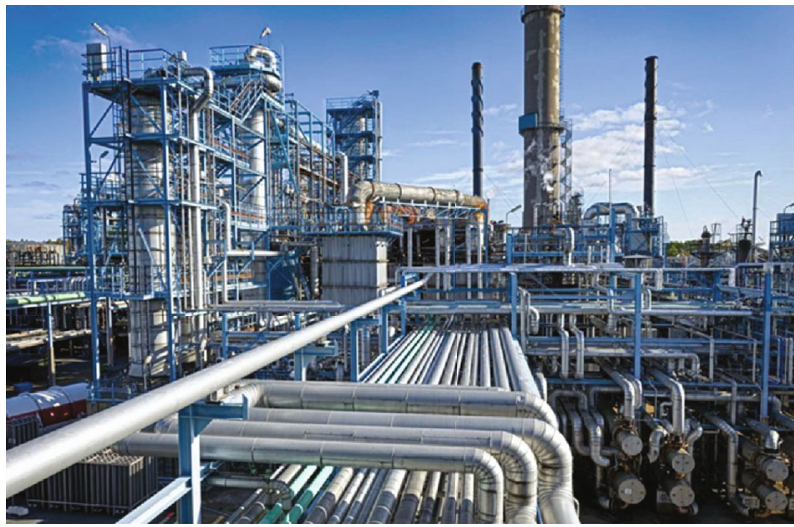

(a)

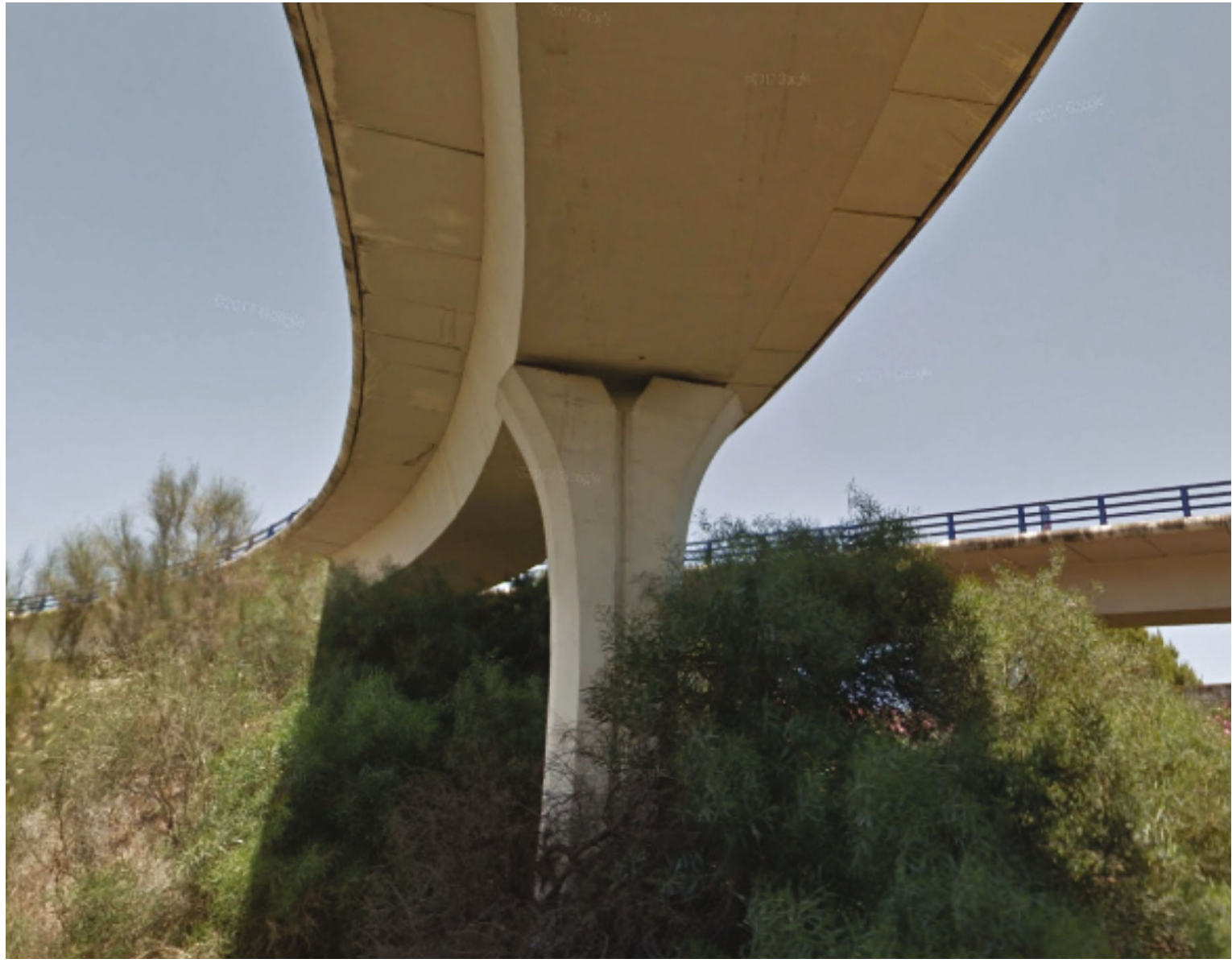

(b)

FIGURE 2: Oil and gas facility (a) and reinforced concrete bridge extracted from [21] (b).

2.2. Modelling. As stated in [23], the dynamics of a multirotor is mainly determined by its mechanical model when it is not operating in the proximity of elements that can modify significantly the rotors' airflow. This section embraces the same assumption for deriving the basic structure of modelling (the characterisation of the aerodynamics will be covered in Section 3), and consequently, the system will be described by means of a detailed mechanical model of the complete multibody system. Kane's method has been used with this purpose since it holds some unique advantages when addressing multibody robotic systems like the ARSLRM. Of the latter, the most remarkable is the derivation of a compact model in first order differential equations that are uncoupled in the generalized speed derivatives as well as the easy computerisation and the computational efficiency of the resulting equations of motion.

The configuration variables selected as system generalized coordinates are the longitudinal $q_{1}$ and vertical $q_{3}$ 


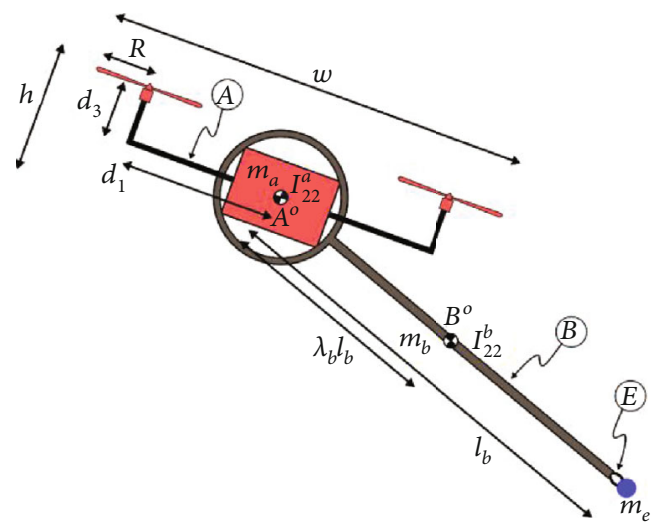

FIgure 3: Aerial Robotic System for Long-Reach Manipulation (ARS-LRM). Geometry and mass distribution.

TABLE 1: ARS-LRM parameters.

\begin{tabular}{lccc}
\hline & Parameter & Value & Units \\
\hline Mass and inertia & $m_{a}$ & 5 & $\mathrm{~kg}$ \\
- & $I_{22}^{a}$ & 0.093 & $\mathrm{~kg} \cdot \mathrm{m}^{2}$ \\
- & $m_{b}$ & 0.3 & $\mathrm{~kg}$ \\
- & $\lambda_{b}$ & 0.5 & - \\
- & $I_{22}^{b}$ & 0.025 & $\mathrm{~kg} \cdot \mathrm{m}^{2}$ \\
- & $m_{e}$ & 0.05 & $\mathrm{~kg}$ \\
Geometry & $w$ & 1.2 & $\mathrm{~m}$ \\
- & $h$ & 0.4 & $\mathrm{~m}$ \\
- & $d_{1}$ & 0.41 & $\mathrm{~m}$ \\
- & $d_{3}$ & 0.2 & $\mathrm{~m}$ \\
- & $R$ & 0.19 & $\mathrm{~m}$ \\
- & $l_{b}$ & 1 & $\mathrm{~m}$ \\
\hline
\end{tabular}

positions of the UAV centre of mass $A^{O}$ in the inertial reference frame $N$, the multirotor pitch angle $q_{5}$, and the joint angle of the robotic arm $q_{7}$ (see Figure 4). Generalized speeds $u_{i}(i=1,3,5,7)$ are defined as

$$
\begin{aligned}
{ }^{N} \mathbf{v}^{A^{O}} & =u_{1} \mathbf{n}_{1}+u_{3} \mathbf{n}_{3}, \\
{ }^{N} \boldsymbol{\omega}^{A} & =u_{5} \mathbf{n}_{2}, \\
{ }^{A} \boldsymbol{\omega}^{B} & =u_{7} \mathbf{n}_{2},
\end{aligned}
$$

where ${ }^{N} \mathbf{v}^{A^{O}}$ is the velocity of the UAV centre of mass $A^{O}$ with respect to the inertial reference frame $N,{ }^{N} \boldsymbol{\omega}^{A}$ is the angular velocity of the aerial platform $A$ with respect to the inertial reference frame $N$, and ${ }^{A} \boldsymbol{\omega}^{B}$ is the angular velocity of the robotic arm $B$ with respect to the aerial platform $A$. Previous equations lead to the following kinematic differential equations:

$$
\left[\begin{array}{llll}
\dot{q}_{1} & \dot{q}_{3} & \dot{q}_{5} & \dot{q}_{7}
\end{array}\right]^{T}=\left[\begin{array}{llll}
u_{1} & u_{3} & u_{5} & u_{7}
\end{array}\right]^{T}
$$

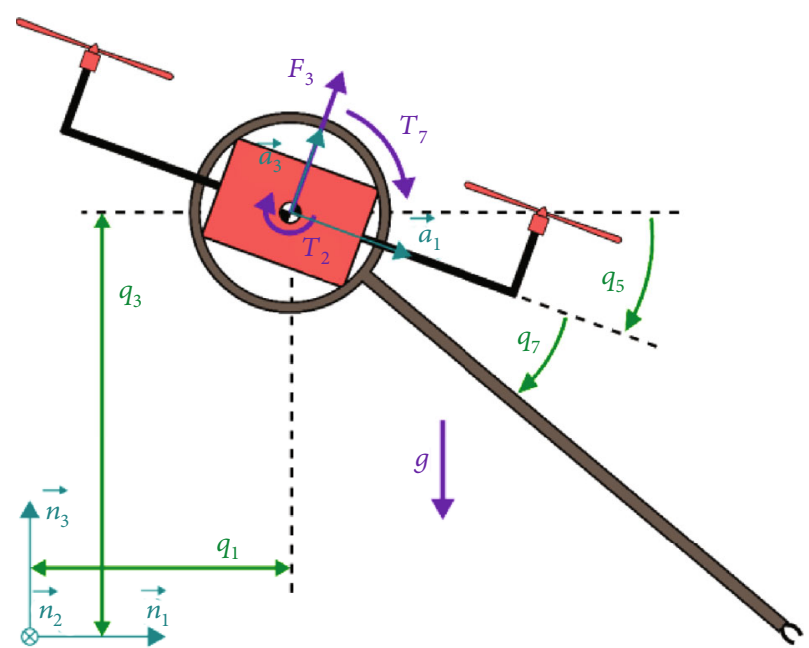

FIgURe 4: Configuration variables (green) and forces and torques applied to the ARS-LRM system (purple).

Regarding forces and torques exerted on the ARS-LRM system (see again Figure 4), the rotors generate a resultant lifting force $F_{3} \mathbf{a}_{3}$ applied to the multirotor centre of mass $A^{O}$ as well as a torque $T_{2} \mathbf{a}_{2}$ applied to the aerial platform $A$. Additionally, the control action governing the manipulator is given by the torque applied to the arm joint $T_{7} \mathbf{a}_{2}$.

Application of Kane's method through MotionGenesis software [24] leads to the following dynamic differential equations for translation and rotation:

$$
\mathbf{A} \cdot\left[\dot{u}_{1} \dot{u_{3}} \dot{u_{5}} \dot{u}_{7}\right]^{T}=\mathbf{B}
$$

where matrix $\mathbf{A}$ is

$\mathbf{A}=\left[\begin{array}{cccc}m_{a}+m_{b}+m_{e} & 0 & -l_{b} \xi_{m, 1} s_{5,7} & -l_{b} \xi_{m, 1} s_{5,7} \\ 0 & m_{a}+m_{b}+m_{e} & -l_{b} \xi_{m, 1} c_{5,7} & -l_{b} \xi_{m, 1} c_{5,7} \\ -l_{b} \xi_{m, 1} s_{5,7} & -l_{b} \xi_{m, 1} c_{5,7} & I_{22}^{a}+I_{22}^{b}+l_{b}^{2} \xi_{m, 2} & I_{22}^{b}+l_{b}^{2} \xi_{m, 2} \\ -l_{b} \xi_{m, 1} s_{5,7} & -l_{b} \xi_{m, 1} c_{5,7} & I_{22}^{b}+l_{b}^{2} \xi_{m, 2} & I_{22}^{b}+l_{b}^{2} \xi_{m, 2}\end{array}\right]$,

matrix $\mathbf{B}$ is

$$
\mathbf{B}=\left[\begin{array}{c}
F_{3} s_{5}+l_{b} \xi_{m, 1} c_{5,7}\left(u_{5}+u_{7}\right)^{2} \\
F_{3} c_{5}-\left(m_{a}+m_{b}+m_{e}\right) g-l_{b} \xi_{m, 1} s_{5,7}\left(u_{5}+u_{7}\right)^{2} \\
T_{2}+l_{b} \xi_{m, 1} c_{5,7} g \\
T_{7}+l_{b} \xi_{m, 1} c_{5,7} g
\end{array}\right],
$$

and $\xi_{m, 1}=m_{e}+\lambda_{b} m_{b}, \xi_{m, 2}=m_{e}+\lambda_{b}^{2} m_{b}, s_{5}=\sin \left(q_{5}\right), c_{5}=$ $\cos \left(q_{5}\right), s_{5,7}=\sin \left(q_{5}+q_{7}\right), c_{5,7}=\cos \left(q_{5}+q_{7}\right)$, and $g=9.81$ $\mathrm{m} / \mathrm{s}^{2}$ is the gravity acceleration.

2.3. Control. A distributed control scheme between the aerial platform and the robotic arm (see Figure 5) has been derived 


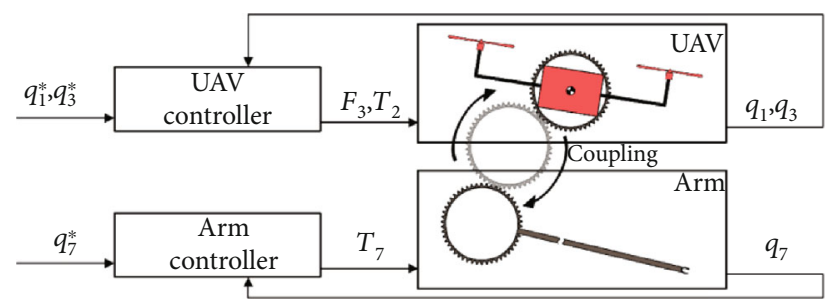

FIGURE 5: Distributed control scheme of the ARS-LRM system.

to provide the ARS-LRM system with the capability of tracking trajectories generated by the motion planning algorithms. The objective is the completion of the simulation environment that will allow the investigation of new planning strategies for the ARS-LRM platform. A standard control structure that makes use of nonlinear control strategies based on model inversion has been selected for this purpose. This choice is considered representative of the average performance offered by the state-of-the-art controllers for this kind of systems.

Regarding the multirotor, the control scheme is inspired by [23] and consists of linearising the system through model inversion and applying PID control laws to the resultant dynamics. In the model inversion mentioned above, the coupling action of the robotic arm is not considered, and hence, it will be treated as a perturbation that must be cancelled by the PID control laws. The underlying principle of control will be the adjustment of the multirotor lifting force vector in order to generate the translational accelerations required to reduce position errors. A general overview of the control scheme is shown in Figure 6(a), where $D_{13}^{-1}, K_{5}^{-1}$, and $D_{5}^{-1}$ blocks represent, respectively, the inversions of the translational dynamics, rotational kinematics, and rotational dynamics.

The control strategy selected for the robotic arm is again based on linearisation through model inversion and PID control, which yields a nonlinear control law capable of commanding the joint position of the manipulator within the allowed rotation rank of $360^{\circ}$. As in the previous case, the coupling action will not be considered in the model inversion, and hence, it will be treated as a perturbation that must be compensated by the control laws. The schematic representation of this approach is shown in Figure 6(b) where $D_{7}^{-1}$ represents the block in charge of inverting the dynamics of the arm and torque $T_{7}$ corresponds to the output signal of the controller.

The parameters of the controllers have been tuned by means of the classic pole assignment method. The selected values constitute a trade-off that guarantees a proper dynamic range while the common mechanical limitations of this kind of systems are not overreached.

\section{Characterisation of Aerodynamic Effects}

The usage of aerial robotic systems for manipulation tasks usually requires that rotors operate in the proximity of surfaces that can affect their airflow. Working under such conditions leads to aerodynamic phenomena that can provoke destabilisations or even crashes. In order to avoid these dan- gerous flight conditions, the compensation of aerodynamic effects should be considered in the design of the controller [19] or taken into account in the trajectory generation process of the motion planner. This paper will adopt the second approach in subsequent sections, but firstly, a characterisation of these effects is required. With that purpose, a test bench that allows the analysis of the thrust behaviour while rotors are operating close to surfaces has been designed.

The test bench consists of an isostatic L-shaped structure that integrates a load cell in its lower endpoint and the rotor whose aerodynamics will be characterised in the distal end of the vertical part (see Figure 7). Additionally, a cylindrical joint $\gamma$ allowing free rotation of the structure has been used to avoid any resultant force in the holder between the ground basis and the corner intersection of the L-shaped structure. An analysis of the force balance in this structure allows the calculation of the rotor thrust $F_{3}$ through the measurement of the reaction force $F_{\mathrm{lc}}$ applied to the load cell. The resultant equation of equilibrium is the following:

$$
F_{3}=F_{\mathrm{lc}} \frac{d_{h}}{d_{v}}
$$

where $d_{h}$ and $d_{v}$ are the dimensions depicted in Figure 7. Regarding the electronics embedded in the structure, the test bench integrates an Arduino Mega 2560 as the data acquisition unit and includes sensors to measure the rotor speed as well as a load cell to collect the measurements of the reaction force $F_{\mathrm{lc}}$. Moreover, the PWM signal commanded to the rotor is monitored at the output using an interruption-based algorithm that runs in the board. The Arduino Mega 2560 is connected through the serial port to a PC with Matlab that offers a graphical interface to process, display, and save all the data collected by the sensors.

As mentioned above, the experimental procedure is based on the measurements of the rotor thrust provided by the test bench. These measurements will be collected in scenarios where an element (see the obstacle in Figure 7) is located close to the rotor (from above, below, or laterally, depending on the particular phenomenon under study, that is, ground effect, ceiling effect, or wall effect). The PWM input signal that governs the rotor speed will be the same in all tests, which in turn implies that the desired rotor thrust will also be the same. However, the measured rotor thrust will change according to the aerodynamic conditions. At the beginning of each experiment, the rotor will receive the constant PWM input during a period of time that guarantees a steady state for the system. From this point on, the measurements registered by the load cell will be filtered conveniently to get the mean value of the measured rotor thrust. The final test result will be the data pair given by the mean value of the thrust and the distance to the element used to disturb the rotor airflow. Several tests are carried out for each distance under study. This makes it possible to obtain statistical characterisations like the standard deviations represented in Figure 8 using an error-bar format.

The experimental results that have been obtained to analyse the ground, ceiling, and wall effects, together with their 


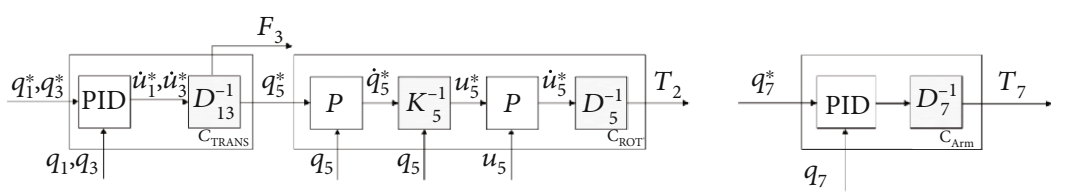

(a)

(b)

FIGURE 6: Block diagrams of the UAV controller (a) and the arm controller (b).
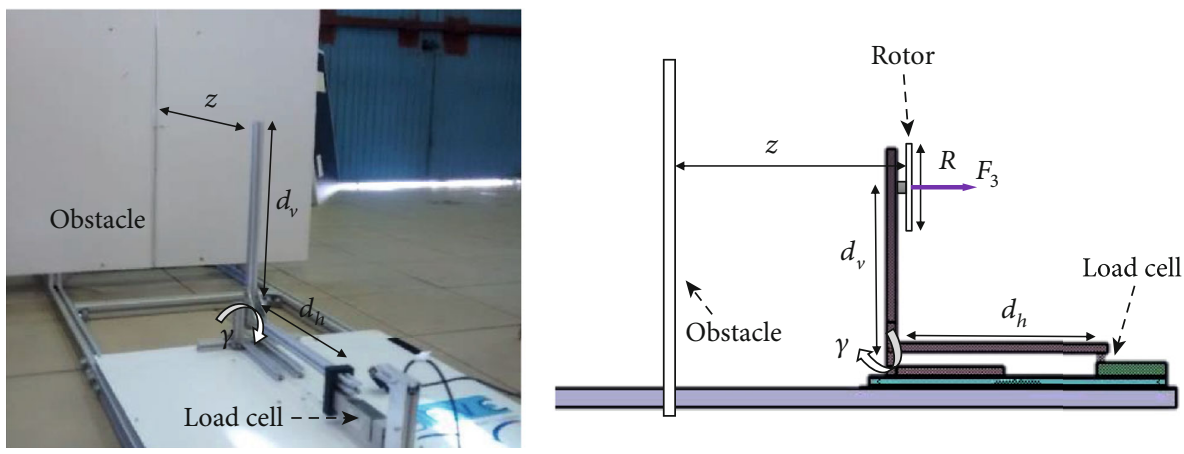

Figure 7: Test bench used for the characterisation of the aerodynamic effects.
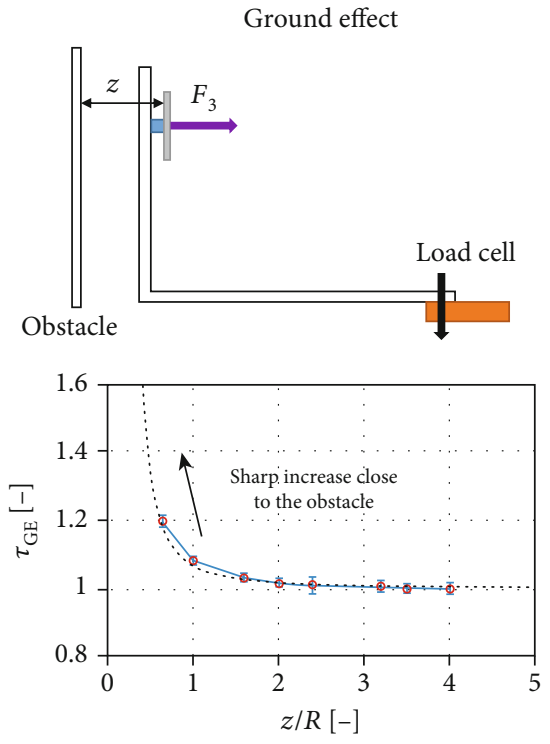

(a)
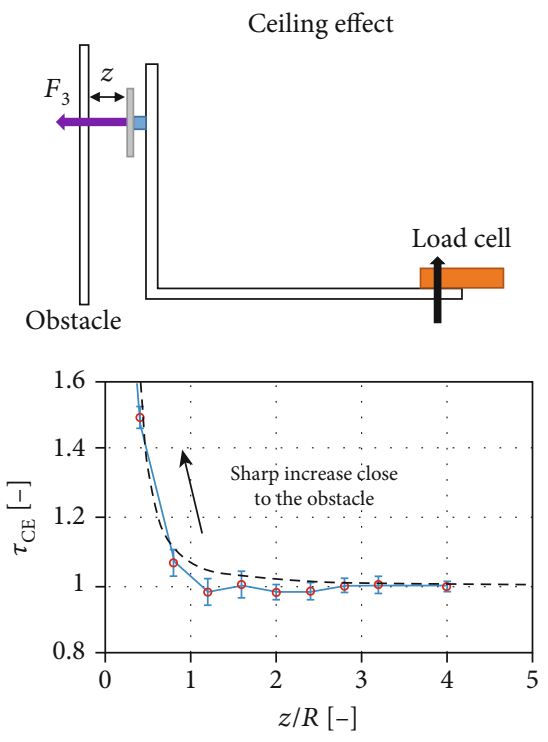

(b)
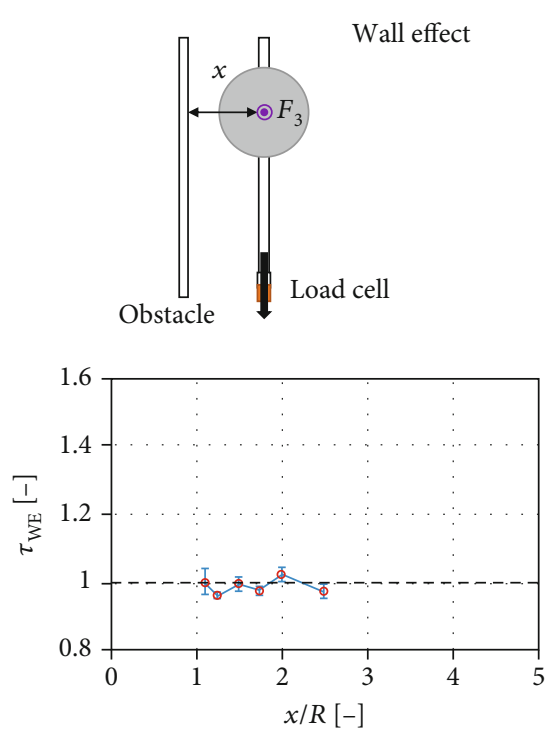

(c)

Figure 8: Characterisation of aerodynamic effects (below) and associated configuration of the test bench (above): ground (a), ceiling (b), and wall (c) effects. Experimental results (red dots and blue error bars) and analytical models (dotted black curve for the theoretical model and dashed black curves for regression models).

associated configurations in the test bench, are shown in Figure 8. In these figures, the aerodynamic modification of the system behaviour is given by $\tau$, a ratio between the lifting force $F_{3}$ in the presence of aerodynamic effects (IAE) and the same force out of the presence of these effects (OAE):

$$
\tau=\frac{F_{3, \mathrm{IAE}}}{F_{3, \mathrm{OAE}}} .
$$

The dependence between $\tau$ and the distance to the surface affecting the rotor airflow is expressed in terms of a nondimensional ratio whose numerator is the distance $x, z$ from the rotor centre to obstacles (see Figure 8) and denominator is the rotor radius, that is, $z / R$ for the ground and ceiling effects or $x / R$ for the wall effect.

The experimental results concerning the ground effect are consistent with the classical approach presented in [25] (dotted black curve in Figure 8(a)). This coincidence 
validates the design of the test bench as well as the experimental procedure. Furthermore, the classical approach previously mentioned is presented in Equation (8) and will be hereinafter adopted to model the ground effect. The derivation of this equation is based on the images method and the assumptions of the linearised potential aerodynamics:

$$
\tau_{\mathrm{GE}}=\frac{1}{1-1 / 16(R / z)^{2}} .
$$

Relative to the ceiling effect, there are no theoretical results in the literature. Alternatively, an experimental approach has been followed to derive an analytical expression:

$$
\tau_{\mathrm{CE}}=\frac{1}{1-1 / k_{1}\left(R /\left(z+k_{2}\right)\right)^{2}},
$$

where the coefficients $k_{1}=6.924$ and $k_{2}=3.782$ have been obtained by the least-square method when minimising the error with the experimental results. The resultant model has been depicted through the dashed black curve in Figure $8(\mathrm{~b})$ for the ceiling effect. The experimental results show that this effect pulls the rotor towards the elements located above when the rotor is working closely. This effect may quickly lead to dangerous flight conditions because it tends to reduce abruptly the safety distance between the rotor and the element above.

Regarding the wall effect, the experimental results shown in Figure 8(c) have demonstrated that it does not have a significant influence on the system. The underlying explanation is that the natural rotor airflow streams from its upper part to its lower part, and therefore, an element located laterally does not produce such a big interference. Consequently, this effect will not be considered in lateral approximations to external elements.

The complete range of effects produced by the aerodynamic phenomena that have been studied in this section can be reflected in 3D maps. To this end, the numerical values corresponding to the different operation points will be computed using the models in Equations (8) and (9). One example of this graphical representation can be seen in Figure 9 for a rectangular-shaped obstacle. These maps will be used in the next section to implement the modifications of the motion planner that enable the Aerodynamics Awareness.

\section{Motion Planner with Dynamics and Aerodynamics Awareness}

This section presents the motion planner (MP) with Dynamics and Aerodynamics Awareness (ADA) derived for the ARS-LRM system. The resultant algorithm will be referred to as MP-ARM-ADA. The acronym ARM stands for the more general classification of aerial robotic manipulators and clarifies that the applicability of the algorithm extends beyond the specific ARS-LRM configuration proposed in this paper for the bridge inspection. In general terms, the basis of

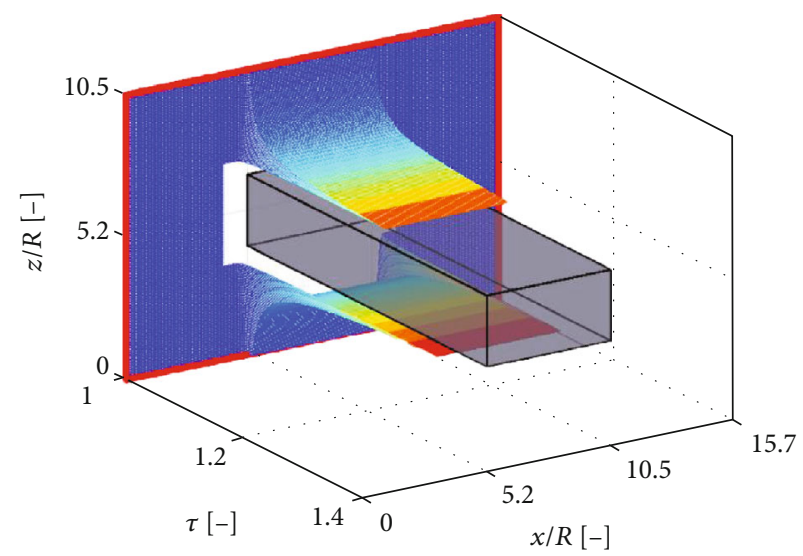

FIGURE 9: 3D map corresponding to the aerodynamic effects associated with a rectangular-shaped obstacle. The colour scale represents the magnitude of the ratio $\tau$ of aerodynamic modification (from lower values in blue to higher values in red) while the white areas are associated with nonflyable regions due to the rotor geometry.

the planning approach (MP-ARM) is built over an RRT* (optimal rapidly exploring random tree [26]) algorithm that has been optimised to exploit the advantages of the specific system under study. Over this optimisation of the basis method, two extensions have been developed to increase the safety of the system when operating in cluttered environments. Firstly, Dynamics Awareness (DA) can be applied to cope with the strong coupling between the aerial platform and the robotic arm. Secondly, Aerodynamics Awareness (ADA) for robust operation close to surfaces involving aerodynamic effects can then be applied. The following subsections develop properly both concepts.

4.1. Fundamentals of Planner Operation. The operation of the MP-ARM planner that constitutes the foundation for subsequent extensions (DA and $\mathrm{ADA}$ ) is based on an integrated exploration of the search space for both subsystems of the ARS-LRM. To this end, the configuration variables of the aerial platform - with the exception of pitch angle $q_{5}$ that will be directly governed by the controller within a bounded range to guarantee smooth movements - and the robotic arm are considered jointly within the planning algorithm (see the configuration variables in Figure 4). This integrated strategy offers higher resolution in the planner operation since equivalent configurations in terms of final effector positions can be differentiated according to the positions of both the multirotor and the robotic arm. The latter makes it possible to achieve wider and safer operating conditions and constitutes a remarkable difference to previous contributions in the field of aerial manipulators where the planning problem was addressed in a decoupled way or only considering the position of the end effector.

Regarding the operation basis for the extension of the search tree, an RRT*-based algorithm (see Algorithm 1) that optimises energy and time performance has been developed. Although the proposed algorithm poses certain structural similarities to the well-known RRT* approach, most of the intermediate functionalities have been customised to deal 


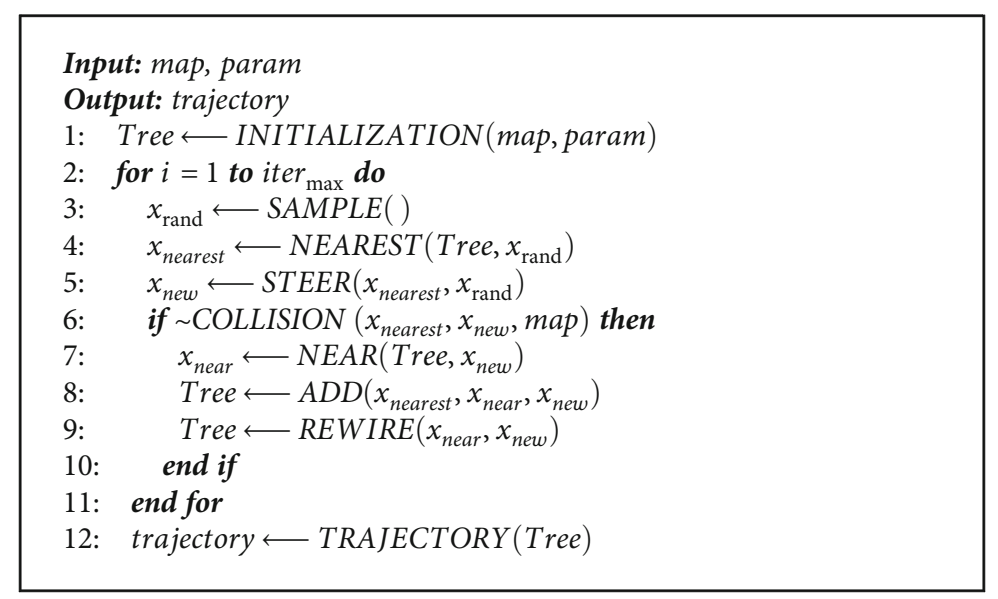

Algorithm 1. Motion Planner for Aerial Robotic Manipulation (MP-ARM).

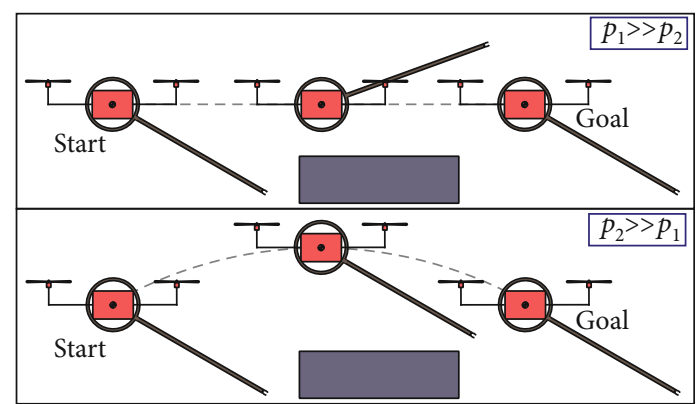

FIGURE 10: Effect of the weighting parameters $p_{1,2}$ on the cost function $\mathrm{CF}=f\left(p_{1,2}\right) \cdot p_{1} \gg p_{2}$ prioritises trajectories in which the energy consumption of the UAV is minimum while $p_{2} \gg p_{1}$ prioritises trajectories in which the energy consumption of the manipulator is minimum.

with the aerial manipulator under study. For instance, Figure 10 illustrates how the two weighting parameters $p_{1,2}$ included in cost function $\mathrm{CF}=f\left(p_{1,2}\right)$ allow the prioritisation of certain movements in the ARS-LRM system. The complete set of these specific derivations have been properly described in a previous contribution [9].

4.2. Dynamics Awareness. The basic approach presented in the previous subsection guarantees planned trajectories that are collision free as well as efficient in terms of execution time and energy consumption. However, the strong dynamical coupling that exists in the ARS-LRM system between the multirotor and the robotic arm requires further attention since it provokes considerable differences between planned and executed trajectories. This is a consequence of the controller limitations to compensate the perturbations associated with the aforementioned couplings. The collision risk introduced by these differences is especially critical for cluttered environments like industrial sites or civil constructions. Figure 11 illustrates an example of the undesired dynamic effects that can arise in such scenarios.

In order to overcome this undesired influence of the dynamical coupling, the inclusion of Dynamics Awareness

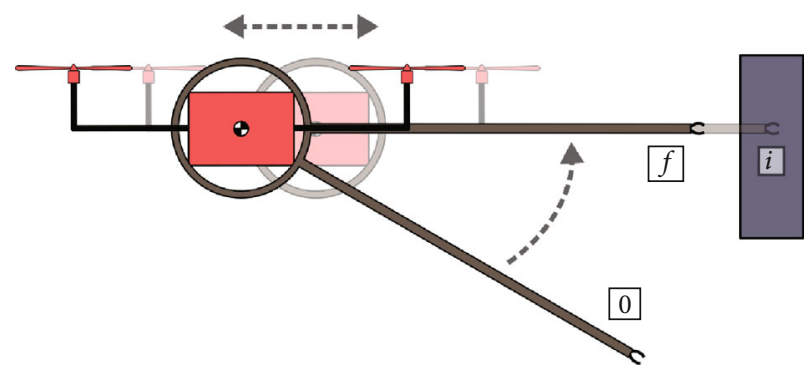

FIGURE 11: ARS-LRM system moving the robotic arm from initial position 0 to final position $f$. The UAV oscillation produced in the intermediate states of this manoeuvre (shaded intermediate position $i$ ) provokes a collision with the obstacle.

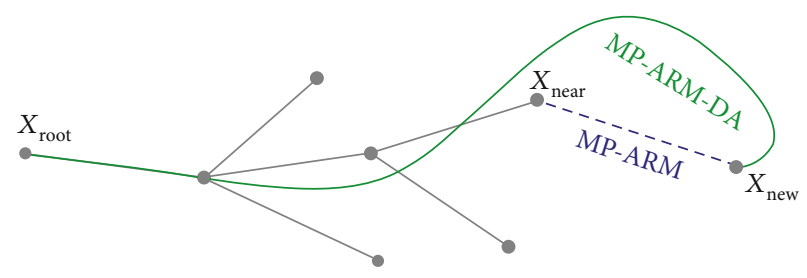

FIGURE 12: Operation basis of the MP-ARM-DA algorithm.

(DA) in the MP-ARM planning algorithm is required. To this end, the expansion of the search tree is based on the behaviour of the controlled system, which means that collision detection is calculated through closed-loop simulations of the controlled system (green solid line in Figure 12) instead of simply using geometrical interpolation between states (blue dashed line in Figure 12). This guarantees that the resultant planned trajectories are both free of obstacles and compatible with the dynamic constraints of the ARSLRM system. The approach considered for the dynamical analysis of the tree extension is based on a root-tocandidate validation. Thus, not only the dynamical feasibility of the new possible branch reaching the candidate node is analysed but also the complete path from the tree root. For more details about this extension of the basis algorithm, please refer to previous work of the authors [10]. 


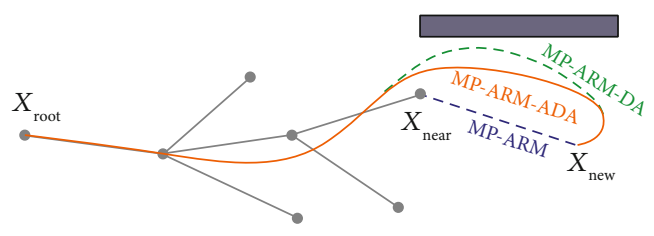

(a)

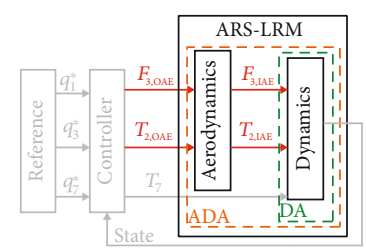

(b)

FIgURE 13: Operation basis of the MP-ARM-ADA algorithm (a) and closed-loop scheme required to perform the simulations that give support to the Aerodynamics Awareness (b). The terms highlighted in red correspond to the variables employed to model the Aerodynamics Awareness.

4.3. Aerodynamics Awareness. When the ARS-LRM system moves autonomously in cluttered environments, the Dynamics Awareness becomes an essential feature for safe operation. However, this mechanism could not be enough for robust obstacle avoidance in some scenarios. As stated in Section 3, aerodynamic phenomena such as the ground effect or the ceiling effect associated with the operation of a multirotor in the proximity of surfaces can modify its aerodynamic forces and, as a consequence, might provoke destabilisations or even crashes. For this reason, in order to avoid these unsafe conditions, this section proposes the inclusion of Aerodynamics Awareness (ADA) within the motion planning process.

Similar to the Dynamics Awareness approach, the principle of operation of the Aerodynamics Awareness mechanism is based on ensuring collision-free trajectories through the closed-loop simulation of the controlled ARS-LRM system. However, in this new approach, not only the dynamics of the system have been considered for the expansion of the search tree but also its aerodynamics. Figure 13(a) schematises the operation basis of the enhanced algorithm (MP-ARM-ADA) in contrast with the MP-ARM and MP-ARM-DA versions. In this figure, the difference between the trajectories computed by MP-ARM-DA and MP-ARM-ADA is the additional safety distance with respect to the grey surface that Aerodynamics Awareness suggests. Otherwise, the controlled system would not be able to compensate for the ceiling effect and might provoke a collision. Additionally, Figure 13(b) depicts the complete closed-loop scheme required to perform the simulations that give support to the Aerodynamics Awareness concept. As can be seen, the MP-ARM-ADA algorithm makes use of an aerodynamic model whose input is given by the control signals commanded by the controller $\left(F_{3, \mathrm{OAE}}, T_{2, \mathrm{OAE}}\right)$ in terms of the corresponding PWM signals and whose output is the force and torque that actually govern the movement of the system as a consequence of aerodynamic effects $\left(F_{3, \mathrm{IAE}}, T_{2, \mathrm{IAE}}\right)$.

In order to derive the block Aerodynamics introduced in Figure 13(b), the force and torque commanded by the controller $\left(F_{3, \mathrm{OAE}}, T_{2, \mathrm{OAE}}\right)$ must be broken down into the contributions of the different rotors. This will pave the way for the application of the equations derived in Section 3 to characterise the aerodynamic effects of an individual rotor. The aforementioned decomposition can be implemented as follows:

$$
\begin{aligned}
& F_{3, i}=F_{3, i}^{\mathrm{L}}+F_{3, i}^{\mathrm{R}}, \\
& T_{2, i}=d_{1}\left(F_{3, i}^{\mathrm{L}}-F_{3, i}^{\mathrm{R}}\right), \quad i=\mathrm{IAE}, \mathrm{OAE},
\end{aligned}
$$

where superscripts $\mathrm{L}$ and $\mathrm{R}$ make reference to the left and right rotors in Figure 4. Additionally, the longitudinal $q_{1}^{\mathrm{L}}$, $q_{1}^{\mathrm{R}}$ and vertical $q_{3}^{L}, q_{3}^{R}$ position of each rotor is also required to calculate the value of the aerodynamic phenomena at a certain operation point:

$$
\begin{aligned}
& q_{1}^{\mathrm{L}}=q_{1}-d_{1} \cos \left(q_{5}\right)+d_{3} \sin \left(q_{5}\right), \\
& q_{3}^{\mathrm{L}}=q_{3}+d_{1} \sin \left(q_{5}\right)+d_{3} \cos \left(q_{5}\right), \\
& q_{1}^{\mathrm{R}}=q_{1}+d_{1} \cos \left(q_{5}\right)+d_{3} \sin \left(q_{5}\right), \\
& q_{3}^{\mathrm{R}}=q_{3}-d_{1} \sin \left(q_{5}\right)+d_{3} \cos \left(q_{5}\right) .
\end{aligned}
$$

Using these positions as an entry point in the $3 \mathrm{D}$ maps computed in Section 3, the aerodynamic ratio $\tau$ between the actual thrust that governs the platform movement (IAE) and the thrust commanded by the controller (OAE) can be obtained. Consequently, the lifting force of each rotor in the presence of aerodynamic effects is given by

$$
F_{3, \mathrm{IAE}}^{j}=\tau\left(q_{1}^{j}, q_{3}^{j}\right) F_{3, \mathrm{OAE}}^{j}, \quad j=\mathrm{L}, \mathrm{R} .
$$

The combined usage of Equations (10) and (12) allows the final calculation of the total force and torque $F_{3, \text { IAE }}, T_{2 \text { IAE }}$ that are really exerted upon the platform.

\section{Application Scenario: Bridge Inspection}

This paper proposes the realistic application of bridge inspection as use case to evaluate the motion planner presented in the previous section. With the means currently available, the application scenario of crack detection in reinforced concrete bridges like the one presented in Figure 2(b) requires visual inspection by qualified human operators. This inspection usually demands the usage of scaffolding or cranes in hard-to-reach and high-altitude locations, which endangers human lives. For this reason, this paper suggests the usage 


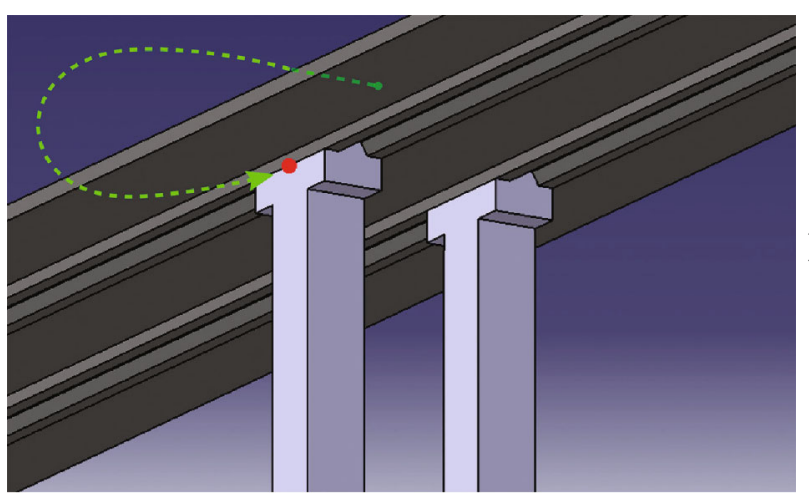

(a)

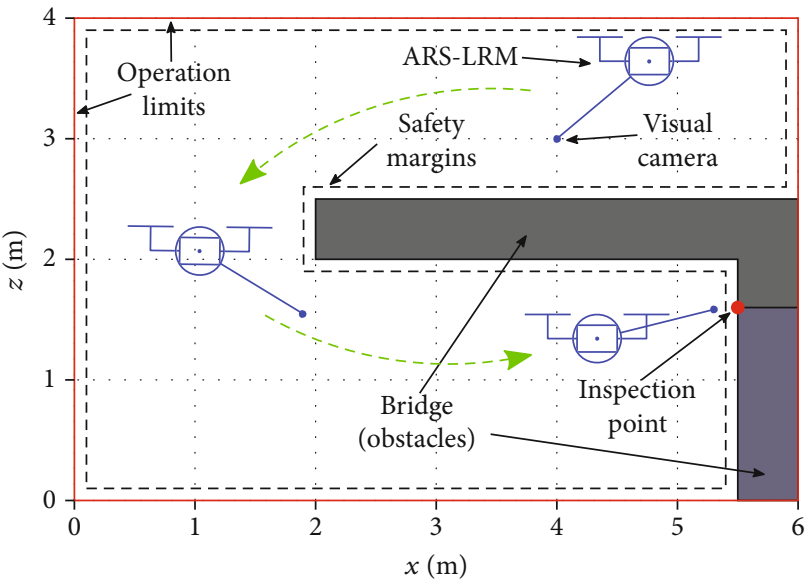

(b)

FIGURE 14: Application scenario given by a bridge inspection task: simulated environment (a) and scheme of the operation (b).

of the ARS-LRM system as an alternative option that avoids human risks and minimises the operational costs.

In order to illustrate the benefits of the ARS-LRM system in the use case under consideration, the simulated environment given by Figure 14(a) will be assumed in this section. In this scenario, the system has to perform a visual inspection of the junction point between the deck and one pillar of the bridge (marked with a red point in the figure). To this end, a visual camera will be integrated as end effector in the long-reach arm of the ARS-LRM system.

The inspection plan will be the following. The aerial system will start the operation from the top part of the bridge and will navigate around the deck until reaching the position where the inspection point is reachable for the end effector. This operation is schematised in Figure 14(b), where the solid red line denotes the operational limits and the dashed black line the safety margins with respect to the obstacles whose violation would be considered as a collision.

The aerodynamic challenges of this scenario arise because the most efficient trajectories for the inspection task under consideration demand that the ARS-LRM system flies close to the bridge surfaces. The latter makes this scenario suitable to establish a first validation of the MP-ARM-ADA algorithm. The expectation is that the resulting trajectories are as efficient as possible in terms of energy consumption but without endangering the integrity of the aerial platform because of the aerodynamic phenomena. In order to characterise these effects, the approach presented in Section 3 has been followed to compute the 3D map of aerodynamic effects corresponding to the application scenario proposed in this section (see Figure 15).

\section{Validation Results}

This section analyses the simulations corresponding to the application of the motion planners presented in Section 4 to the inspection scenario previously described. This validation analysis has been structured around two main phases. As a first step, the performance of the algorithms previously

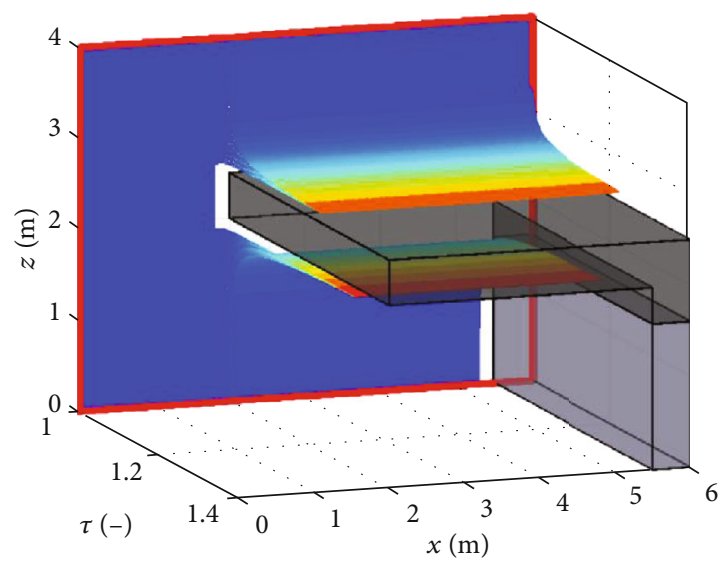

FIGURE 15: 3D map of aerodynamic effects for the bridge scenario.

developed by the authors along their work-line with aerial long-reach manipulators, that is, the MP-ARM and the MP-ARM-DA algorithms presented in $[9,10]$, respectively, has been evaluated. The latter have revealed their limitations in the inspection scenario under consideration because of their lack of Aerodynamics Awareness. As a second step, a complete analysis of the MP-ARM-ADA performance has also been addressed. The analysis of these results allows it to be concluded that the Aerodynamics Awareness is required to generate both efficient and safe trajectories when the ARS-LRM system operates close to elements that can modify significantly the rotor airflow.

In general terms, the validation tests have been carried out following the same sequence. Firstly, the planning algorithm under analysis has been executed to generate the motion plan. Then, the resultant plan has been provided to the controlled ARS-LRM system. The objective is therefore to analyse not only the planned trajectories but also the closed-loop simulations of the controlled system when following the commanded trajectories. In fact, the latter will be considered the most representative information to establish the performance of the different algorithms. 


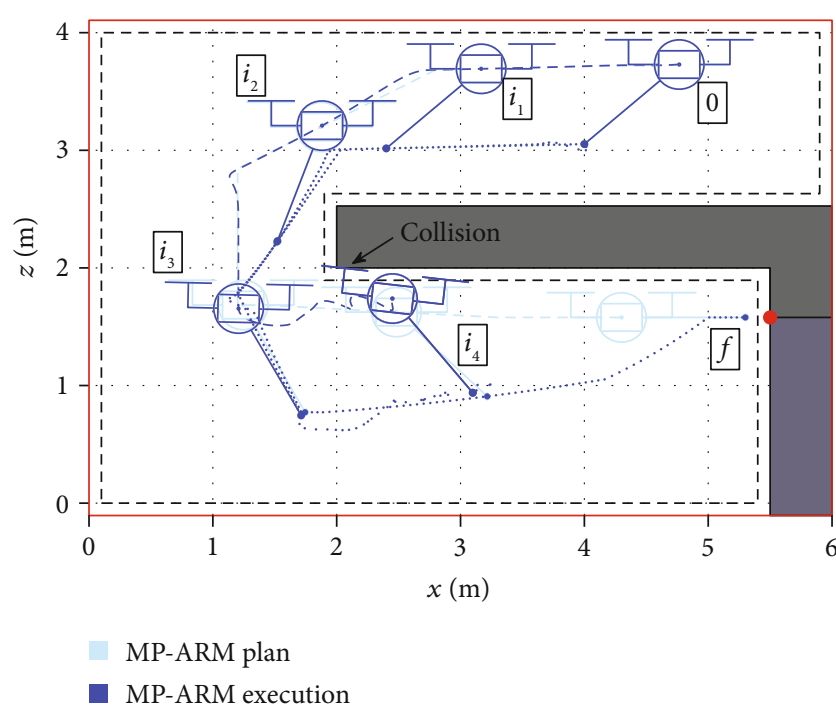

Figure 16: Snapshot diagram corresponding to the closed-loop execution (dark blue) of the trajectory planned with the MP-ARM algorithm (light blue).

The simulation work has been performed in a MatlabSimulink framework that provides the graphical evolution of the system variables together with the corresponding virtual reality animations. Both graphical outputs will be used throughout this section to illustrate the obtained results. Additionally, intuitive snapshot diagrams have also been included. In these diagrams, the dashed and dotted coloured lines represent, respectively, the planned (light colours) and the simulated (dark colours) movements of the multirotor centre of mass as well as the positioning of the end effector. In contrast, the snapshots themselves only cover some intermediate configurations to illustrate intuitively the qualitative behaviour of the ARS-LRM system. These representative configurations are time-ordered by identifying labels that go from the initial position (label 0) to the final position (label $f$ ) passing through a set of intermediate positions (labels $i_{1}, i_{2}, i_{3}, i_{4}$ ).

6.1. Results Using the MP-ARM Algorithm. Figures 16 and 17 show the closed-loop simulation results when the controlled ARS-LRM system (dark blue) tracks the trajectory planned with the MP-ARM motion planner (light blue). Additionally, a complete video animation can be found in [27]. While the planned trajectory can be considered efficient and collision-free, the attempt to execute such optimal trajectory provokes serious inconveniences due to the aerodynamic phenomena. More precisely, since the controlled ARS-LRM system is commanded to navigate close to the bottom surface of the bridge deck (the shortest trajectory to the final inspection pose shown in Figure 16), this implies flying in the presence of the associated ceiling effect. The latter produces disturbances that the controller is not able to reject. Consequently, the system becomes unstable until finally the rear rotors collide with the bridge.
6.2. Results Using the MP-ARM-DA Algorithm. As in the previous section, Figures 18 and 19 depict the simulation results when the controlled ARS-LRM system (dark green) tracks a trajectory generated by the MP-ARM-DA motion planner (light green). Similarly, the corresponding video animation can be found in [28]. Although in this case the planner takes into account the dynamics of the system within the planning process, the disturbances associated with the ceiling effect lead again the ARS-LRM system to collide with the bridge deck in the closed-loop simulation. The only remarkable difference is that the collision instant is delayed. This is a consequence of the addition of Dynamics Awareness since the planner is now aware of the collision risk produced by the states close to the safety margins (overshooting associated with these states that can be observed in the dynamic simulations). As a result, the MP-ARM-DA planner discards those states. However, this improvement is not enough to operate safely in this application scenario since the collision eventually takes place.

6.3. Results Using the MP-ARM-ADA Algorithm. In order to conclude with the simulation sequence, Figures 20 and 21 show the results when a trajectory generated by the MPARM-ADA motion planner (light orange) is commanded to the controlled ARS-LRM system (dark orange). [29] includes the full video animation of the simulation. In contrast to MP-ARM and MP-ARM-DA, the enhanced MPARM-ADA algorithm avoids navigating through the hazardous area close to the bridge. Thanks to the Aerodynamics Awareness, the planner discards these problematic states during the planning process and explores alternatives that lead to the most efficient trajectories but within the area of safe operation. To this end, the MP-ARM-ADA algorithm also takes advantage of the long-reach capabilities of the ARS-LRM system. They make it possible for the aerial platform to reach the inspection point from positions that maintain a wider safety margin with respect to the elements causing the aerodynamic phenomena.

In order to endorse the validity of previous conclusions about the MP-ARM-ADA algorithm, a wider set of simulations has been carried out. More particularly, this algorithm has been extensively executed in the inspection bridge scenario to obtain a sample of 50 planned trajectories, all of these leading to collision-free executions. The same extensive approach has been followed with the MP-ARM and MPARM-DA algorithms, and only one instance of the MPARM-DA led to a collision-free trajectory. This analysis reinforces the capacity of the MP-ARM-ADA method to plan efficient trajectories that at the same time ensure safe conditions of operation.

Moreover, Figure 22 presents the analysis of two general metrics of the MP-ARM-ADA planned trajectories; these are the length of both the UAV and the arm trajectories. The central red line is the median while the edges of the box are the $25^{\text {th }}$ and $75^{\text {th }}$ percentiles. The whiskers extend to the most extreme values obtained in the simulations. As can be seen for the length of the UAV trajectory, there exists small variability around the median while the worst and best cases are relatively close to each other. At the same time, the 

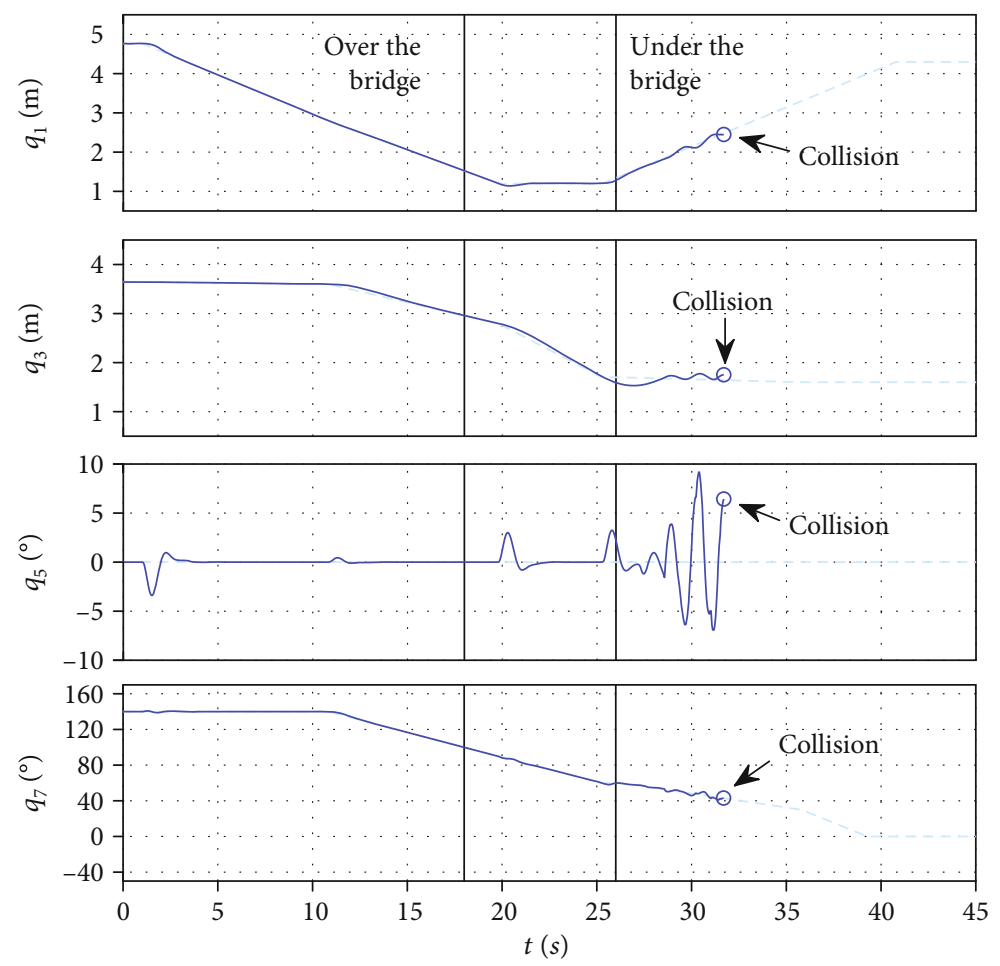

MP-ARM plan

- MP-ARM execution

FIGURE 17: Trajectory planned with the MP-ARM algorithm (dashed light blue) and executed by the controlled ARS-LRM system (solid dark blue).

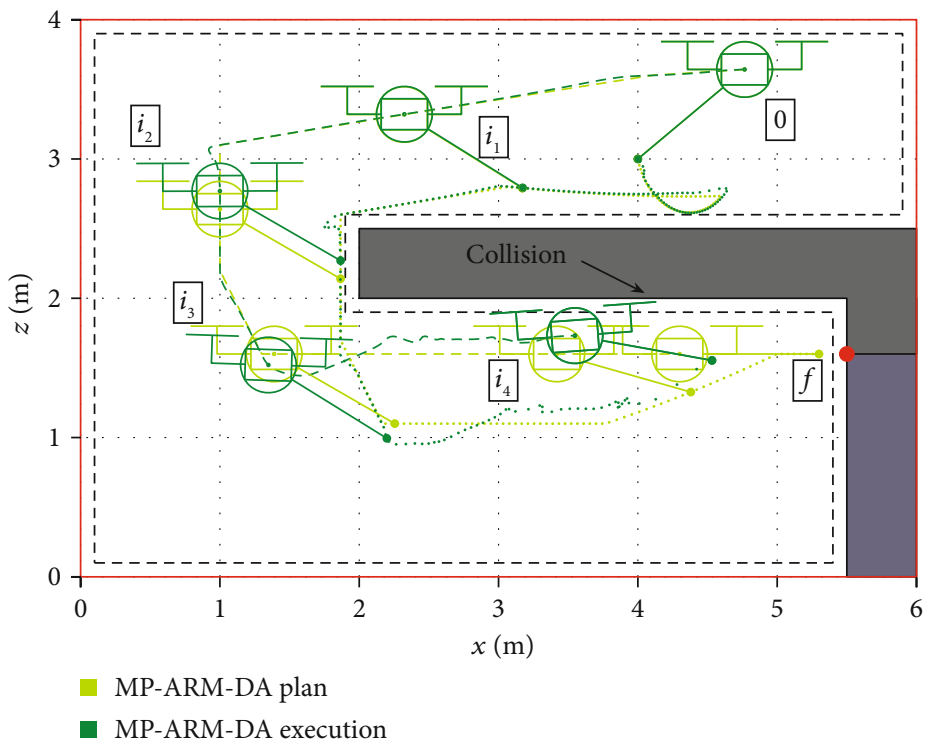

FIGURE 18: Snapshot diagram corresponding to the closed-loop execution (dark green) of the trajectory planned with the MP-ARM-DA algorithm (light green).

angular displacement of the robotic arm is the same for all the simulations (the minimum distance between the initial and final poses) because the planner always computes the optimal trajectory for this subsystem. These results thus reveal a deterministic behaviour of the algorithm in the performance indices under consideration, which can also be considered a desirable feature in critical operation conditions like the proximity of the bridge. 

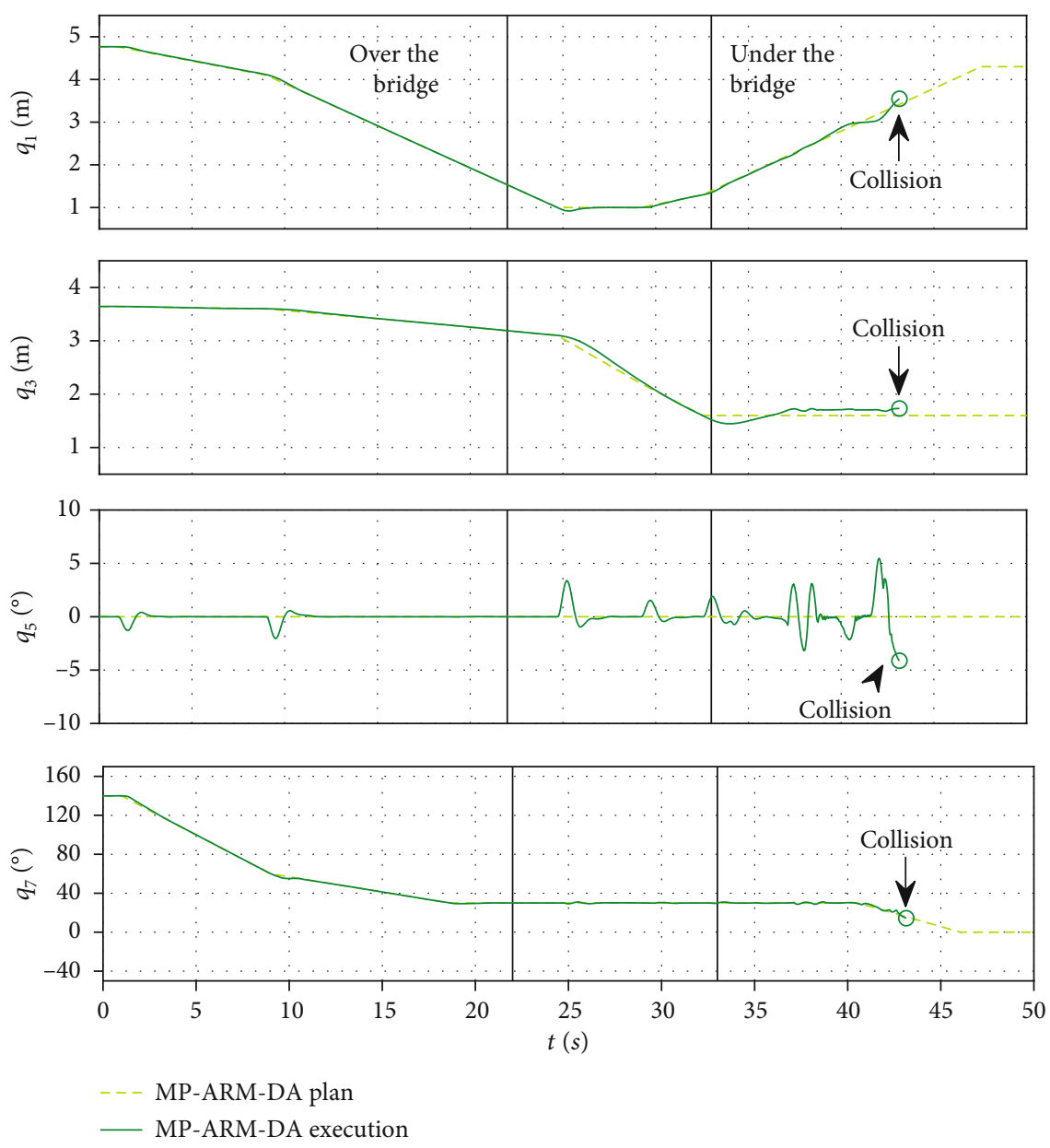

FIGURE 19: Trajectory planned with the MP-ARM-DA algorithm (dashed light green) and executed by the controlled ARS-LRM system (solid dark green).

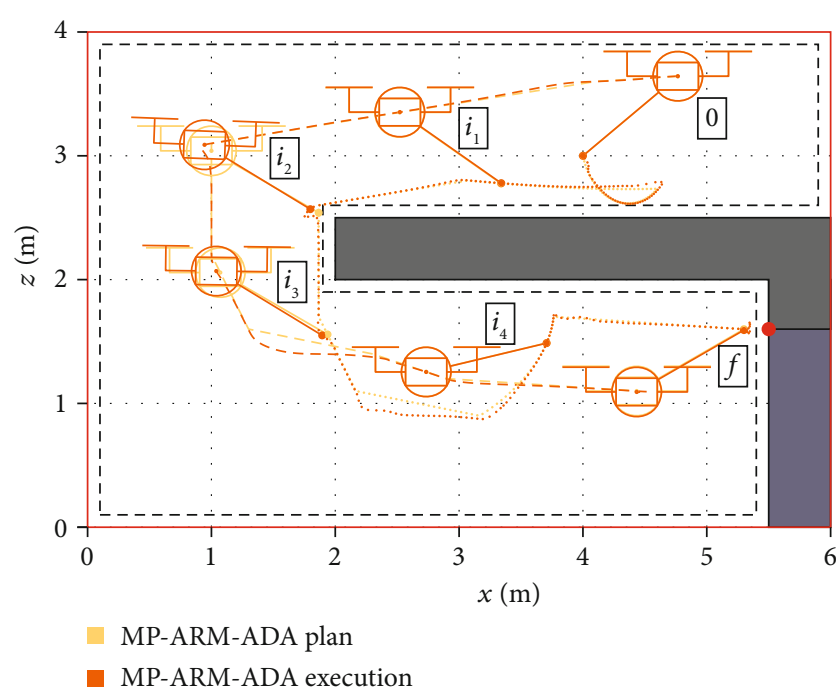

Figure 20: Snapshot diagram corresponding to the closed-loop execution (dark orange) of the trajectory planned with the MPARM-ADA algorithm (light orange).

\section{Conclusions}

This work extends the state of the art in the field of motion planning for aerial long-reach manipulators. In particular, an Aerodynamics Awareness (ADA) mechanism that allows the consideration of aerodynamics within the trajectory generation process has been developed. The validation tests presented in the final part of the paper have demonstrated that the application of this novel algorithm MP-ARM-ADA becomes essential when an aerial robot has to operate in the proximity of large surfaces that can affect the rotor airflow.

The validation has been carried out in a realistic scenario: the inspection of the pillar of a bridge to find potential cracks. There exists, however, a wider range of applications that can benefit from this Aerodynamics Awareness. Inspection and maintenance of industrial scenarios like oil and gas facilities are a clear example since the existence of large pipe arrays and bulky tanks can provoke aerodynamic interaction.

The design of the proposed system, an Aerial Robotic System for Long-Reach Manipulation (ARS-LRM), has also been demonstrated to be very convenient for this kind of operations. Firstly, its capability for multidirectional movement widens significantly the inspection workspace of the 

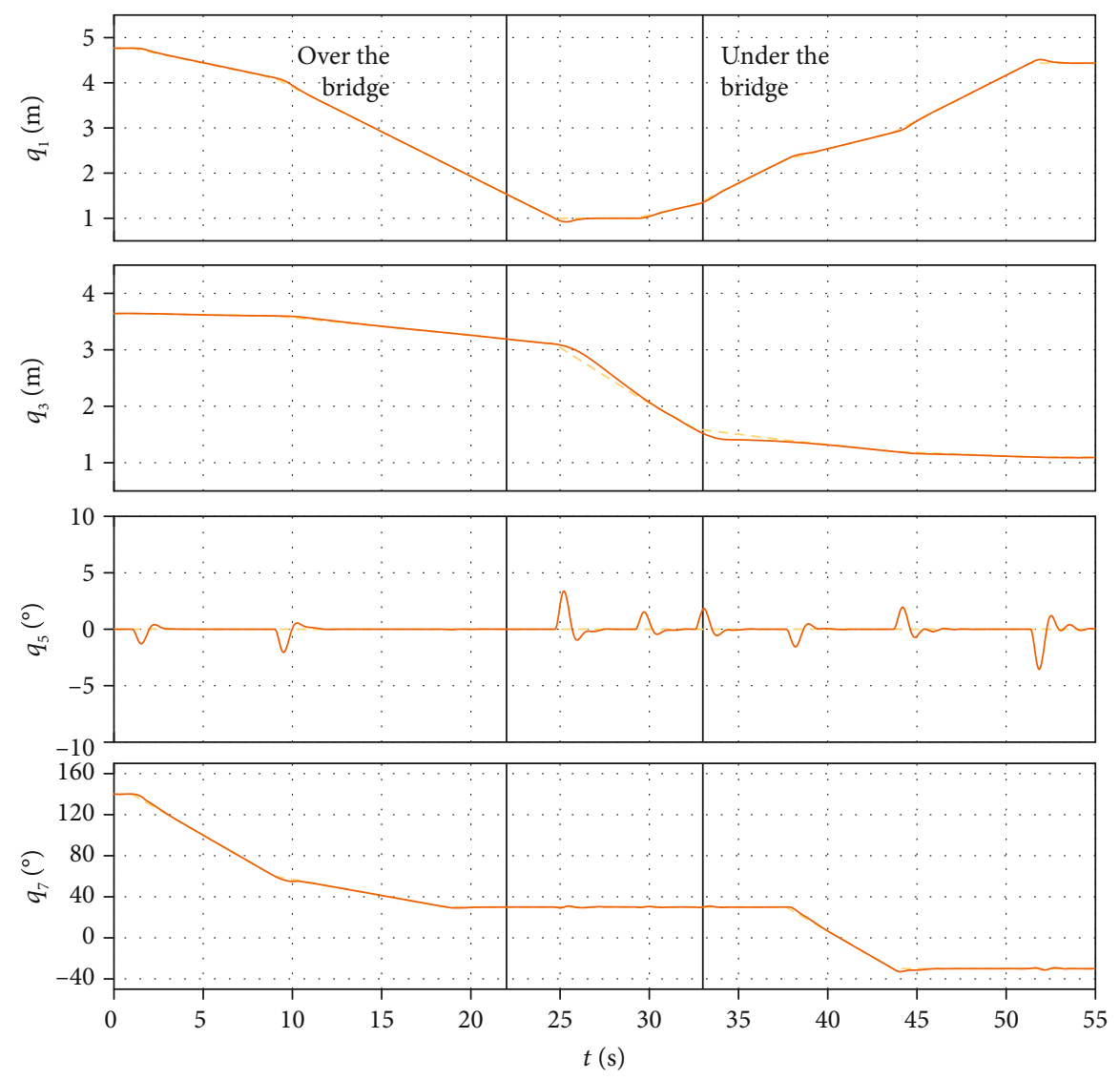

MP-ARM-ADA plan

- MP-ARM-ADA execution

FIGURE 21: Trajectory planned with the MP-ARM-ADA algorithm (dashed light orange) and executed by the controlled ARS-LRM system (solid dark orange).
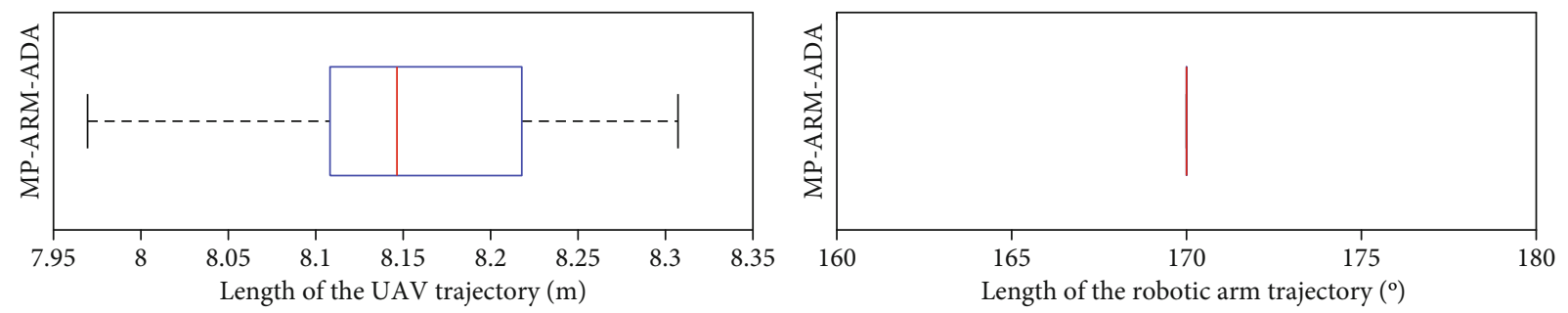

FIgURE 22: Statistical analysis for the set of 50 trajectories planned with the MP-ARM-ADA algorithm.

robotic arm, which in turn extends the range of positions from which the aerial platform can perform the inspection task. The latter allows the selection of the safest operation conditions for the aerial system. Secondly, the long-reach feature increments the safety distance between rotors and inspected areas. This last characteristic is even more important when the inspected areas include elements that can affect the rotor airflow, as is the case in the motivating scenario for this research work.

Future extensions of this work will focus on enlarging the range of aerodynamic effects considered by the motion planner. This will pave the way to inspect typical elements of those industrial scenarios previously mentioned, such as pipe arrays or chimneys. In this new context, the application of ADA would allow the detection of leaks in pipes, the measurement of chimney thicknesses, or the visual inspection of control valves, among others. Experimental validation will be accomplished in the same facilities considered for previous demonstration works developed by the authors, which includes bridges, cement plants, and refineries [7].

\section{Data Availability}

The data used to support the findings of this study are available from the corresponding author upon request. 


\section{Conflicts of Interest}

The authors declare that there is no conflict of interest regarding the publication of this paper.

\section{Acknowledgments}

This work has been supported by the European Projects HYFLIERS and RESIST, funded by the Horizon 2020 research and innovation programme of the European Commission under grant agreements 779411 and 769066, the ARM-EXTEND project funded by the Spanish Ministerio de Economia y Competitividad (DPI2017-89790-R), the ARTIC project funded by the Spanish Ministerio de Ciencia, Innovación y Universidades (RTI2018-102224-B-I00), and the Spanish Ministries MICINN (FPI program 2018) and MECD (FPU program).

\section{References}

[1] D. Mellinger, Q. Lindsey, M. Shomin, and V. Kumar, "Design, modeling, estimation and control for aerial grasping and manipulation," in Intelligent Robots and Systems (IROS), 2011 IEEE/RSJ International Conference on, pp. 2668-2673, San Francisco, CA, USA, September 2011.

[2] A. Suarez, A. E. Jimenez-Cano, V. Vega, G. Heredia, A. Rodriguez-Castaño, and A. Ollero, "Lightweight and human-size dual arm aerial manipulator," in Unmanned Aircraft Systems (ICUAS), 2017 International Conference on, pp. 1778-1784, Miami, FL, USA, USA, June 2017.

[3] K. Kondak, F. Huber, M. Schwarzbach et al., "Aerial manipulation robot composed of an autonomous helicopter and a 7 degrees of freedom industrial manipulator," in Robotics and Automation (ICRA), 2014 IEEE International Conference on, pp. 2107-2112, Hong Kong, China, June 2014.

[4] A. E. Jimenez-Cano, J. Braga, G. Heredia, and A. Ollero, "Aerial manipulator for structure inspection by contact from the underside," in Intelligent Robots and Systems (IROS), 2015 IEEE/RSJ International Conference on, pp. 1879-1884, Hamburg, Germany, October 2015.

[5] A. E. Jimenez-Cano, G. Heredia, and A. Ollero, "Aerial manipulator with a compliant arm for bridge inspection," in Unmanned Aircraft Systems (ICUAS), 2017 International Conference on, pp. 1217-1222, Miami, FL, USA, USA, June 2017.

[6] P. Božek, M. A. Al Akkad, P. Blištan, and N. I. Ibrahim, "Navigation control and stability investigation of a mobile robot based on a hexacopter equipped with an integrated manipulator," International Journal of Advanced Robotic Systems, vol. 14, no. 6, pp. 1-13, 2017.

[7] M. A. Trujillo, J. R. Martinez-de Dios, C. Martin, A. Viguria, and A. Ollero, "Novel aerial manipulator for accurate and robust industrial NDT contact inspection: a new tool for the oil and gas inspection industry," Sensors, vol. 19, no. 6, p. $1305,2019$.

[8] A. Caballero, M. Bejar, and A. Ollero, "On the use of velocity adaptation to outperform the motion planning with dynamics awareness in aerial long-reach manipulators with two arms," in 2018 International Conference on Unmanned Aircraft Systems (ICUAS), pp. 1125-1133, Dallas, TX, USA, June 2018.

[9] A. Caballero, M. Bejar, A. Rodriguez-Castaño, and A. Ollero, "Motion planning for long reach manipulation in aerial robotic systems with two arms," in 2017 European Conference on Mobile Robots (ECMR), pp. 1-7, Paris, France, September 2017.

[10] A. Caballero, M. Bejar, A. Rodriguez-Castaño, and A. Ollero, "Motion planning with dynamics awareness for long reach manipulation in aerial robotic systems with two arms," International Journal of Advanced Robotic Systems, vol. 15, no. 3, p. 172988141877052, 2018.

[11] A. Caballero, A. Suarez, F. Real et al., "First experimental results on motion planning for transportation in aerial longreach manipulators with two arms," in Intelligent Robots and Systems (IROS), 2018 IEEE/RSJ International Conference on, Madrid, Spain, October 2018.

[12] R. Ragel, I. Maza, F. Caballero, and A. Ollero, "Comparison of motion planning techniques for a multirotor UAS equipped with a multi-joint manipulator arm," in Research, Education and Development of Unmanned Aerial Systems (RED-UAS), 2015 Workshop on, pp. 133-141, Cancun, Mexico, November 2015.

[13] M. H. Korayem, H. R. Nohooji, and A. Nikoobin, "Path planning of mobile elastic robotic arms by indirect approach of optimal control," International Journal of Advanced Robotic Systems, vol. 8, no. 1, pp. 10-20, 2017.

[14] M. H. Korayem and A. M. Shafei, "A new approach for dynamic modeling of n-viscoelastic-link robotic manipulators mounted on a mobile base," Nonlinear Dynamics, vol. 79, no. 4, pp. 2767-2786, 2015.

[15] S. M. LaValle and J. J. Kuffner Jr., "Randomized kinodynamic planning," The International Journal of Robotics Research, vol. 20, no. 5, pp. 378-400, 2016.

[16] E. Koyuncu and G. Inalhan, “A probabilistic B-spline motion planning algorithm for unmanned helicopters flying in dense $3 \mathrm{D}$ environments," in Intelligent Robots and Systems (IROS), 2008 IEEE/RSJ International Conference on, pp. 815-821, Nice, France, September 2008.

[17] C. Richter, A. Bry, and N. Roy, "Polynomial trajectory planning for aggressive quadrotor flight in dense indoor environments," in Robotics Research, pp. 649-666, Springer, 2016.

[18] L. Danjun, Z. Yan, S. Zongying, and L. Geng, "Autonomous landing of quadrotor based on ground effect modelling," in 34th Chinese Control Conference (CCC), pp. 5647-5652, Hangzhou, China, July 2015.

[19] P. J. Sanchez-Cuevas, G. Heredia, and A. Ollero, "Characterization of the aerodynamic ground effect and its influence in multirotor control," International Journal of Aerospace Engineering, vol. 2017, 17 pages, 2017.

[20] P. J. Sanchez-Cuevas, G. Heredia, and A. Ollero, "Multirotor UAS for bridge inspection by contact using the ceiling effect," in Unmanned Aircraft Systems (ICUAS), 2017 International Conference on, pp. 767-774, Miami, FL, USA, USA, June 2017.

[21] Google Maps, https://www.google.com/maps/@37.4071952,-5 $.9489918,3 \mathrm{a}, 90 \mathrm{y}, 24.23 \mathrm{~h}, 109.91 \mathrm{t} / \mathrm{d}$ a t a $=$ ! $3 \mathrm{~m} 6$ ! 1 e 1 ! $3 \mathrm{~m} 4$ ! $1 \mathrm{~s}$ C $2 \mathrm{H} \mathrm{g} 4 \mathrm{r} \mathrm{L} \mathrm{X} \mathrm{e} \mathrm{Y} \mathrm{W}$ zI7hC1jjFg!2e0!7i13312!8i6656.

[22] AEROARMS Project, AErial RObotic system integrating multiple ARMS and advanced manipulation capabilities for inspection and maintenance, 2019, https://aeroarms-project.eu/.

[23] K. Kondak, M. Bernard, N. Meyer, and G. Hommel, "Autonomously flying VTOL-robots: modeling and control," in Robotics and Automation (ICRA), 2007 IEEE International Conference on, pp. 736-741, Roma, Italy, April 2007. 
[24] Motiongenesis Kane 5.x, http://www.motiongenesis.com/.

[25] I. Cheeseman and W. Bennett, The Effect of Ground on a Helicopter Rotor in Forward Flight, 1955.

[26] S. Karaman and E. Frazzoli, "Sampling-based algorithms for optimal motion planning," The international journal of robotics research, vol. 30, no. 7, pp. 846-894, 2011.

[27] Simulation results using the MP-ARM algorithm. $2 D$ animation, https://youtu.be/IlMszdx2MwA.

[28] Simulation results using the MP-ARM-DA algorithm. $2 D$ animation, https://youtu.be/awbBWJ7mJRo.

[29] Simulation results using the MP-ARM-ADA algorithm. 2D animation, https://youtu.be/FzG9uvcjjbs. 